\title{
EBIS Au beam at RHIC
}

\author{
S.Y. Zhang
}

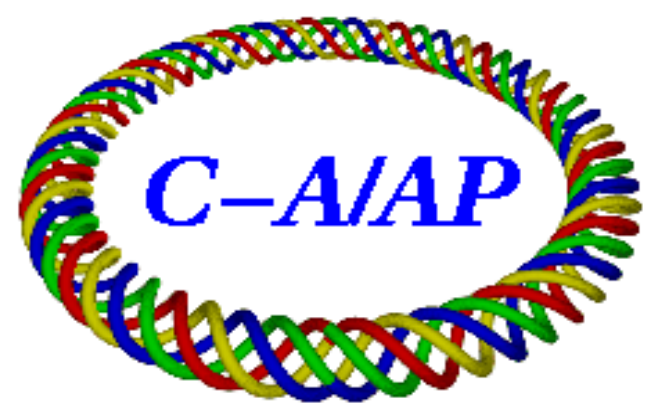

\section{Collider-Accelerator Department Brookhaven National Laboratory \\ Upton, NY 11973}

Notice: This document has been authorized by employees of Brookhaven Science Associates, LLC under Contract No. DE-AC02-98CH10886 with the U.S. Department of Energy. The United States Government retains a nonexclusive, paid-up, irrevocable, world-wide license to publish or reproduce the published form of this document, or allow others to do so, for United States Government purposes. 


\title{
EBIS Au Beam at RHIC
}

\author{
S.Y. Zhang
}

\begin{abstract}
With the EBIS beam and current setting in injector chain, the $\mathrm{Au}$ beam at RHIC injection is different from the Tandem beam. The transverse and longitudinal emittance of the EBIS Au beam at RHIC is presented in this note. For further improvements of EBIS and injector settings, this can be used to compare or to justify. Also, based on Run 2012 conditions, the luminosity optimization in RHIC is of interest to study.
\end{abstract}

\section{Introduction}

In 2012, the EBIS beam is used for $\mathrm{U}-\mathrm{U}$ run and $\mathrm{Cu}-\mathrm{Au}$ run. In $\mathrm{Cu}-\mathrm{Au}$ run (Run12), 4 Booster loads are merged into 1 RHIC bunch with the $\mathrm{Au}$ intensity of $1.6 \times 10^{9}$ ions. The intensity is close to previous $\mathrm{Au}-\mathrm{Au}$ runs with the Tandem as the ion source, e.g., Run 2011 (Run11), but the transverse and longitudinal emittance at RHIC is not the same.

A rather strong dependence of the transverse emittance on bunch intensity is observed in $\mathrm{Cu}-\mathrm{Au}$ run. It is not straightforward to get the emittance dependence on $\mathrm{Au}$ bunch intensity in a $\mathrm{Cu}-\mathrm{Au}$ run, but some estimate can be made.

In Run12, the longitudinal emittance of $\mathrm{Au}$ beam at the RHIC injection is about twice as large as that with the Tandem beam. The subsequent longitudinal emittance growth during the ramp is weaker than Run11. Moreover, the longitudinal emittance growth with high intensity is not stronger than the low intensity ramp in Run12. This is explained by the IBS simulation with the larger initial transverse emittance for higher intensities. The fitting of the initial transverse emittance is approximately agreeable with the estimate of the transverse emittance dependence on intensity. 
The longitudinal emittance at the RHIC injection, along with the transverse emittance and its dependence on intensity, suggest that the EBIS and the injector's settings might be improved. The improvement can be compared with, and perhaps justified by, the Run12 conditions presented in this note.

The RHIC luminosity optimization can be studied under the current conditions, and also for the improved EBIS and injector's settings.

\section{Transverse emittance}

The transverse emittance at the RHIC early collision in $\mathrm{Cu}-\mathrm{Au}$ run is calculated from the PHENIX ZDC, and called the ZDC emittance. With low intensity it seems comparable with the previous $\mathrm{Au}-\mathrm{Au}$ runs. However, along with the increase of bunch intensity, both $\mathrm{Cu}$ and $\mathrm{Au}$, the emittance is clearly rising. This is not observed in previous runs using the Tandem as the ion source.

In Fig.1a, the ZDC emittance is compared with the $\mathrm{Au}$ bunch intensity from $0.8 \times 10^{9}$ to $1.6 \times 10^{9}$ ions. The $\mathrm{Au}$ bunch intensity is at the RHIC injection, and the ZDC emittance is at the early collision. Shown are 61 fills from 16902 to 17027.

The transverse emittance dependence on bunch intensity is also seen from the IPM measurement, for both $\mathrm{Cu}$ and $\mathrm{Au}$ and for both horizontal and vertical. In Fig.1b, the horizontal Yellow ( $\mathrm{Au}$ ) beam IPM emittance at the early collision is compared with the Au intensity.

Along with the $\mathrm{Au}$ intensity improvement, the $\mathrm{Cu}$ beam intensity is also increased, but not always in same steps. In Fig.1c, the $\mathrm{Cu}$ bunch intensity is plotted against $\mathrm{Au}$ bunch intensity for each fill in 16902 to 17027.

Assuming that the initial transverse emittance of $\mathrm{Cu}$ and $\mathrm{Au}$ are not too different, one may estimate that with the Au bunch intensity from $0.8 \times 10^{9}$ to $1.6 \times 10^{9}$, the transverse emittance at the RHIC injection is increased from $10 \pi \mu m$ to $20 \pi \mu m$. Similar transverse emittance increase is also estimated for $\mathrm{Cu}$ bunch intensity from $2.5 \times 10^{9}$ to $5 \times 10^{9}$.

The mechanism of the transverse emittance dependence on intensity is under study. In Fig.2, the machine performance from the EBIS exit to RHIC injection is shown for fills 16951 to 16998, where the Au bunch intensity at the RHIC injection is increased from approximately $0.8 \times 10^{9}$ to $1.6 \times 10^{9}$. 

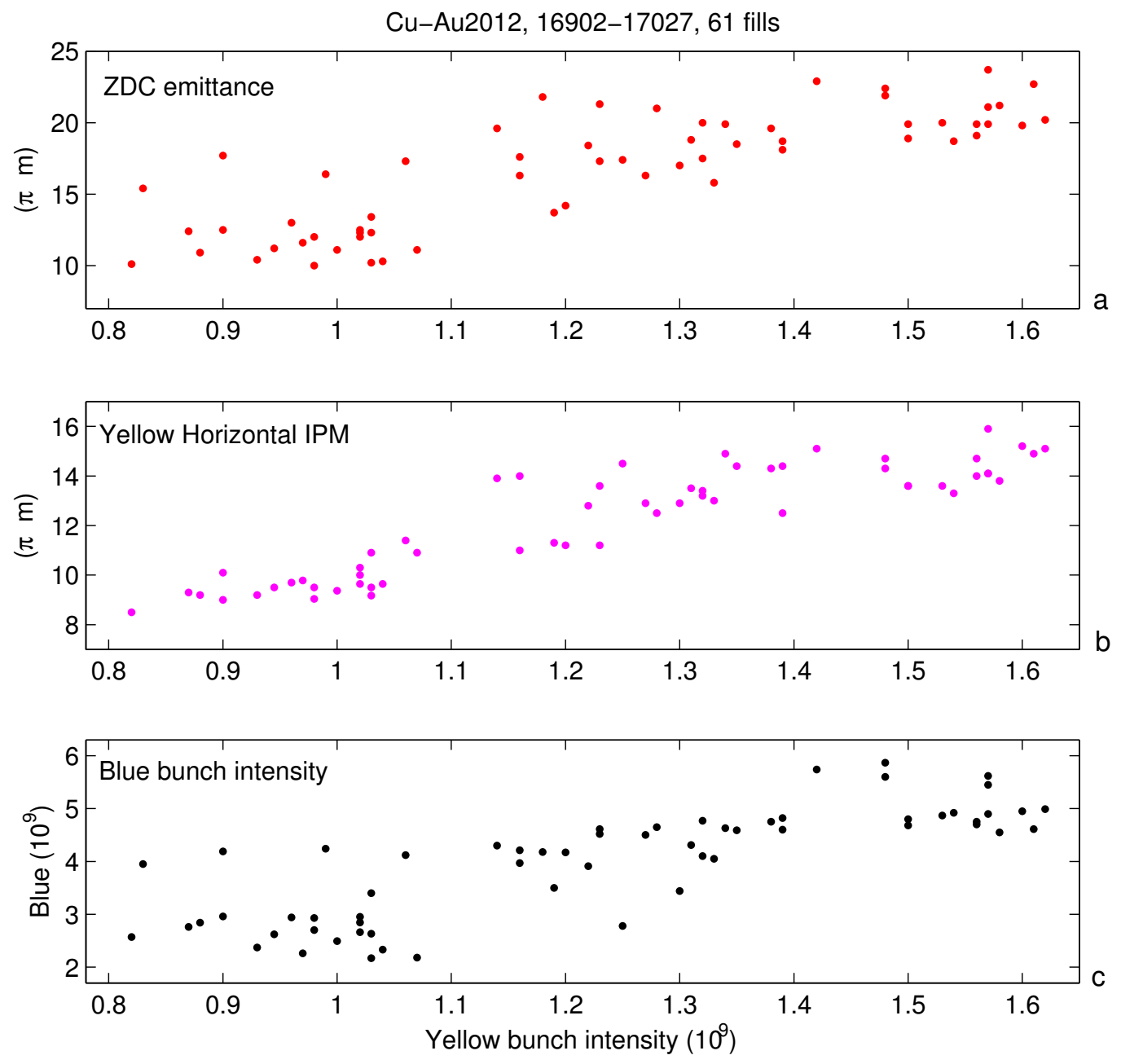

Figure 1: Fills 16902 to 17027, total 61 fills. In Fig.1a, the ZDC emittance, calculated from PHENIX ZDC at the initial collision is compared with the Yellow $(\mathrm{Au})$ bunch intensity, taken at the RHIC injection. In Fig.1b, the Yellow horizontal IPM emittance at the initial collision is compared with the $\mathrm{Au}$ bunch intensity. In Fig.1c, Blue $(\mathrm{Cu})$ bunch intensity is plotted against the Au bunch intensity. 

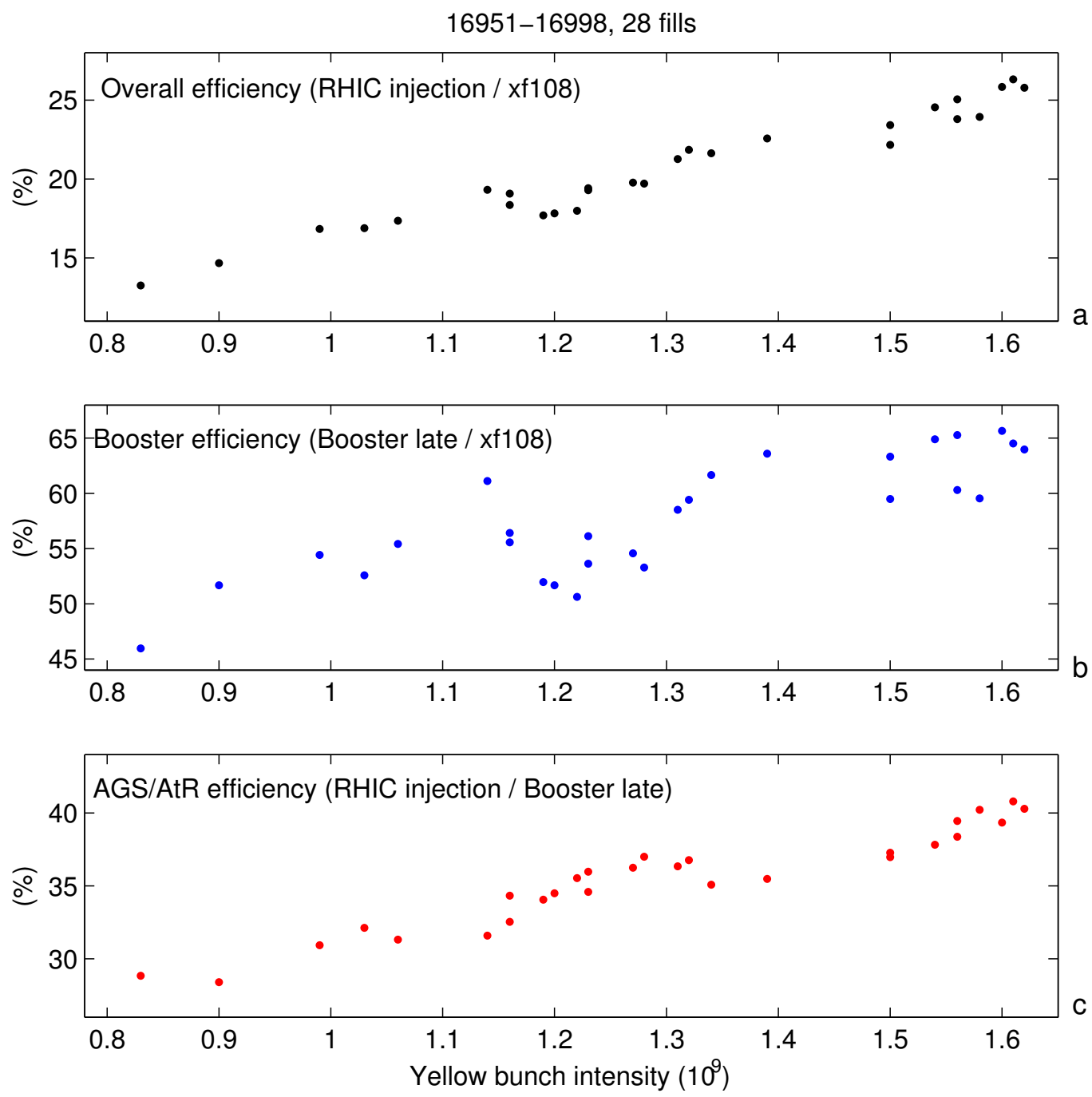

Figure 2: Fills 16951 to 16998 , where the Au bunch intensity at the RHIC injection is increased from $0.8 \times 10^{9}$ to $1.6 \times 10^{9}$. The Booster efficiency includes the Booster injection and ramp. The AGS/AtR efficiency includes the BtA transmission, the AGS ramp, eliminating the baby bunches, and AtR. 
During the entire period from 16951 to 16998, with Au bunch intensity increased from $0.8 \times 10^{9}$ to $1.6 \times 10^{9}$, one may notice:

1. Almost entire RHIC bunch intensity improvement from $0.8 \times 10^{9}$ to $1.6 \times 10^{9}$ can be accounted by the efficiency improvement from xf108 to RHIC injection, as shown in Fig.2a. Therefore, the EBIS beam, judged from the $\mathrm{xf108}$, is almost flat, suggesting that the transverse emittance growth is not related to EBIS.

2. The Booster efficiency (Booster late / xf108) is improved by about $35 \%$ as shown in Fig.2b, which includes the Booster injection and the Booster ramp, with bunch merge.

3. The AGS/AtR efficiency is improved by about $45 \%$ as shown in Fig.2c, where the BtA stripping efficiency is flat at about 55\%, but the AGS ramping with bunch merge, the reducing of 'baby bunches', and the AtR tunning are all improved.

More study is needed to locate the main culprit(s) of the emittance growth, which includes the bunch merges at the Booster and the AGS. The BtA stripping foil's effect (Booster late is increased about 35\% during the period) may also need to clarify.

\section{Longitudinal emittance}

In Run12, the initial longitudinal emittance of $\mathrm{Au}$ beam at the RHIC injection is between $0.5 \mathrm{eVs} / n$ to $0.6 \mathrm{eV} \mathrm{s} / n$, not depending on bunch intensity in general. As the comparison, typical RHIC initial longitudinal emittance with the Tandem beam in Run11 is $0.25 \mathrm{eVs} / n$ to $0.3 \mathrm{eVs} / \mathrm{n}$, also not depending on intensity.

The longitudinal emittance evolution at the RHIC injection and ramp is different for Run11 and Run12:

1. From the beam injection to start ramp, taking at least a few minutes, the longitudinal emittance in Run11 is typically increased from $0.3 \mathrm{eVs} / \mathrm{n}$ to $\geq 0.4 \mathrm{eVs} / \mathrm{n}$. In Run12, the growth is much smaller.

2. In Run12, the longitudinal emittance growth of the higher intensity ramp is not stronger than the low intensity ramp, which is different from Run11.

It seems these can be explained by the factors related to the IBS longitudinal growth rate, 


$$
\tau_{\|}^{-1}=\frac{r_{i}^{2} c N_{b h} \lambda}{8 \beta^{3 / 2} \gamma^{3 / 2} \epsilon_{N, r m s}^{3 / 2}\left\langle\beta_{x}^{1 / 2}\right\rangle \sigma_{s} \delta_{p}^{2}} \propto \frac{N_{b h}}{\epsilon_{N}^{3 / 2} \epsilon_{\ell}^{3 / 2}}
$$

where the first part of the equation can be found in [1]. In the second part, the bunch intensity $N_{b h}$, the normalized transverse emittance $\epsilon_{N}$, and the longitudinal emittance $\epsilon_{\ell}$ are singled out for the effect of the longitudinal growth rate. The relation of the longitudinal emittance $\epsilon_{\ell}$ with the bunch length $\sigma_{s}$ and the momentum spread $\delta_{p}$, i.e.,

$$
\sigma_{s} \delta_{p}^{2} \propto \epsilon_{\ell}^{3 / 2}
$$

can be derived assuming the RF bucket not changed.

Since the initial longitudinal emittance at RHIC in Run12 is twice as large as in Run11, the longitudinal growth rate in Run12 is about $1 / 3$ of Run11, given other conditions the same. This explains, partially, the slower longitudinal emittance growth during the injection in Run12.

If the longitudinal and transverse emittance at the RHIC injection is the same, the initial longitudinal emittance growth rate should be proportional to bunch intensity, according to Equation 1. Along with the time, however, the growth rate with higher intensity beam would be weakened by the faster rising of both $\epsilon_{N}$ and $\epsilon_{\ell}$ (due to the high intensity), therefore, the overall longitudinal emittance growth is less than proportional to bunch intensity. The longer the time, the weaker the intensity effect on the IBS longitudinal emittance growth.

In overall, however, the beam with higher intensity still has larger longitudinal emittance growth, which is observed in Run11 [2].

For higher intensity beam in Run12, the initial transverse emittance is larger. With this effect on the longitudinal growth rate shown in Equation 1, i.e., $1 / \epsilon_{N}^{3 / 2}$, the longitudinal emittance growth with higher intensity in Run12 may not be stronger, after all.

In Fig.3, on the left side, 3 fills in Run12 with different bunch intensities are shown for the observed bunch length and longitudinal emittance during the ramp. On the right side, the IBS simulation under different bunch intensity and initial transverse emittances are shown to compare. In the simulation, the initial transverse emittance is set larger for higher intensity fills. The details are shown in Table 1. 


\begin{tabular}{ccccc}
\hline \hline Fill & & 16951 & 16940 & 16998 \\
\hline Bunch intensity, $N_{b h}$ & $10^{9}$ & 0.73 & 1.25 & 1.51 \\
Initial longitudinal emittance, $\epsilon_{\ell}$ & $e V s / n$ & 0.51 & 0.50 & 0.56 \\
End longitudinal emittance, $\epsilon_{\ell}$ & $e V s / n$ & 0.78 & 0.74 & 0.78 \\
Initial transverse emittance, $\epsilon_{N}$ & $\pi \mu m$ & 9.5 & 14 & 18 \\
\hline \hline
\end{tabular}

Table 1: Fills 16951, 16940, and 16998 in Run12. Shown are bunch intensity and the simulated longitudinal emittance at accramp (initial) and at flatop (end), which is close to the observed. The initial transverse emittance is larger for higher intensity fills in the IBS simulation, which is approximately agreeable with the estimated transverse emittance dependence on the bunch intensity in Run12.

\section{Impact on RHIC luminosity}

In Fig.4, on the left side is the observed bunch length and longitudinal emittance in ramp, for 16086 in Run11 and 16998 in Run12, both fills have bunch intensity about $1.5 \times 10^{9}$. On the right side is the IBS simulation, 16086 of Run11 with the initial transverse emittance of $9 \pi \mu m$, and 16998 of Run12 with $18 \pi \mu m$. In addition to the different initial longitudinal and transverse emittance, the ramp time, the RF voltage, and the lattice are different for Run11 and Run12.

Despite the larger initial value, the longitudinal emittance in Run12 at the full energy is smaller than Run11. There are several reasons:

1. The ramp time in Run12 is reduced from 380 second to 330 second, which is helpful for cutting the total IBS effect in ramp, both longitudinal and transverse.

2. Larger initial longitudinal emittance in Run12 gives rise to lower IBS growth rate, both longitudinal and transverse.

3. Most importantly, the larger initial transverse emittance in Run12 is taking a role on the IBS effect. On the right side of Fig.3, the dashed line shows the IBS growth simulation of 16998 in Run12 with the initial transverse emittance used for Run11, $9 \pi \mu m$. As the result, the longitudinal emittance would be larger than $1 \mathrm{eVs} / n$ at the flatop. 
Green: $16951,0.73 \times 10^{9} /$ bunch Blue:16940,1.25 $\times 10^{9} /$ bunch Red: $16998,1.51 \times 10^{9} /$ bunch
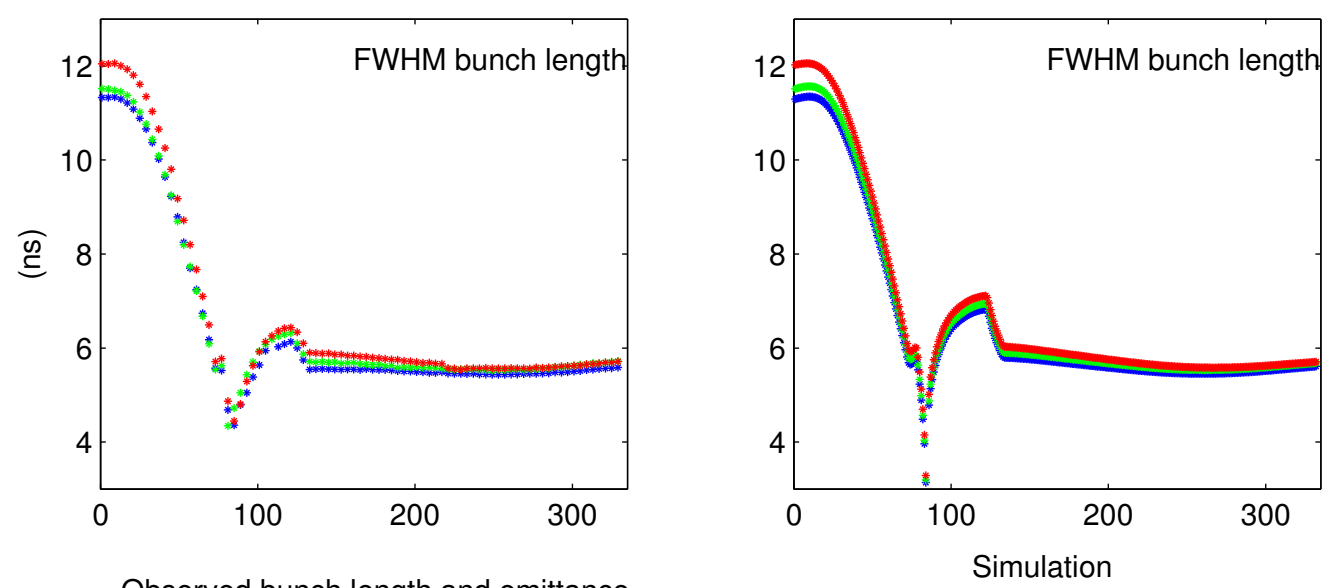

Observed bunch length and emittance

16951:9.5 $\mu \mathrm{m}, 16940: 14 \pi \mu \mathrm{m}, 16998: 18 \pi \mu \mathrm{m}$
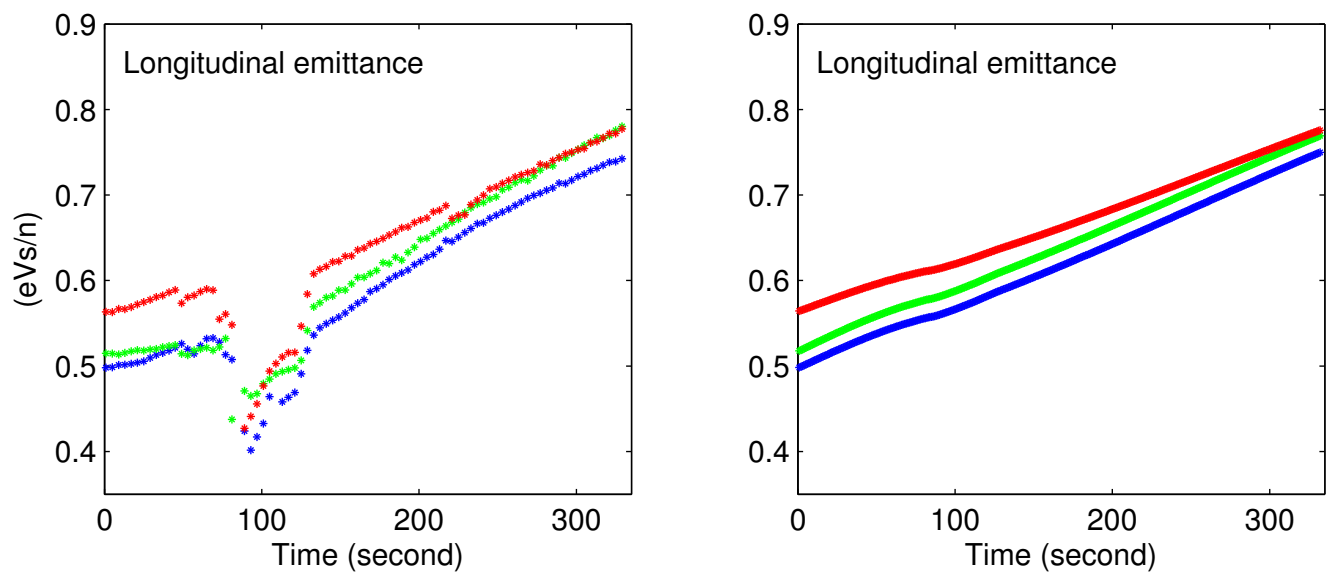

Figure 3: On the left side, the observed FWHM bunch length and the longitudinal emittance during the ramp in Run12 for fills of 16951 with Au bunch intensity of $0.73 \times 10^{9}, 16940$ with $1.25 \times 10^{9}$, and 16998 with $1.51 \times 10^{9}$, are shown. The longitudinal emittance growth of higher intensity beam is not any stronger. On the right side, the IBS simulation is shown, with the initial transverse emittance of 16951 set at $9.5 \pi \mu m, 16940$ at $14 \pi \mu m$, and 16998 at $18 \pi \mu m$. 
Blue: 16086(Run11), $1.50 \times 10^{9} /$ bunch

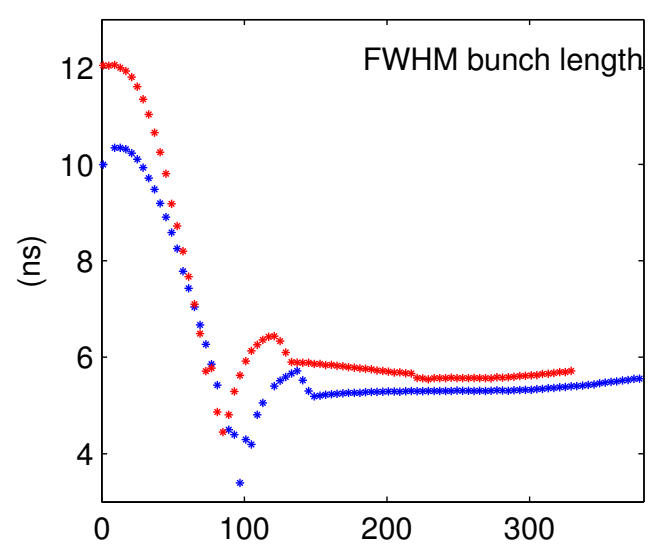

Observed bunch length and emittance

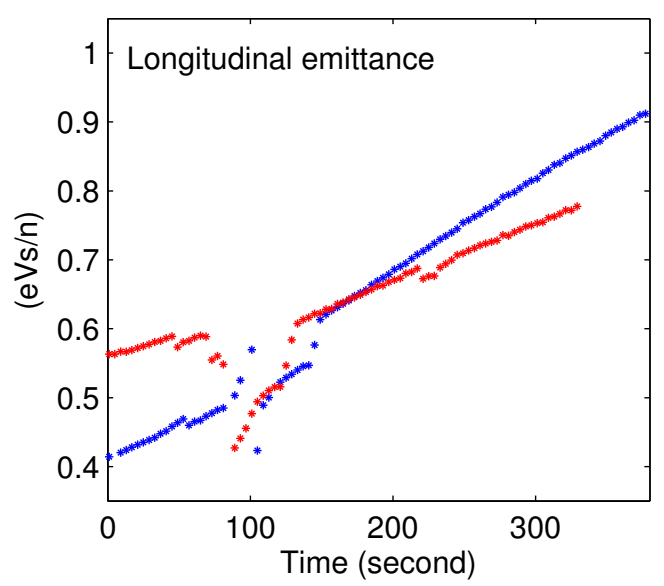

Red: 16998(Run12), $1.51 \times 10^{9} /$ bunch

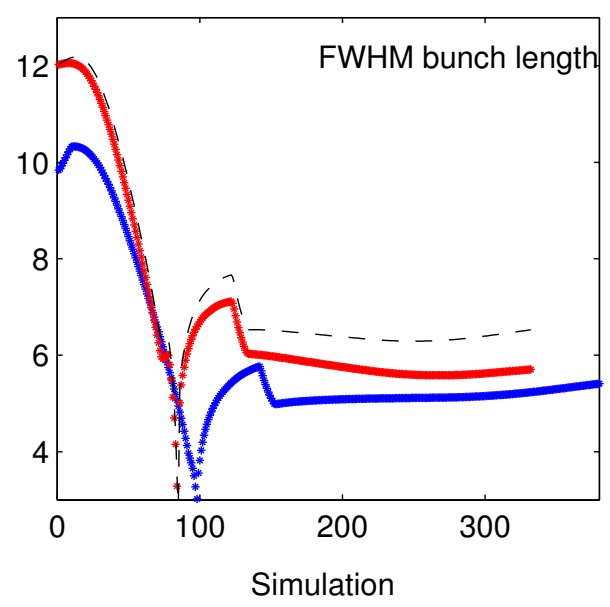

16086: $9 \pi \mu \mathrm{m}, 16998: 18 \pi \mu \mathrm{m}$

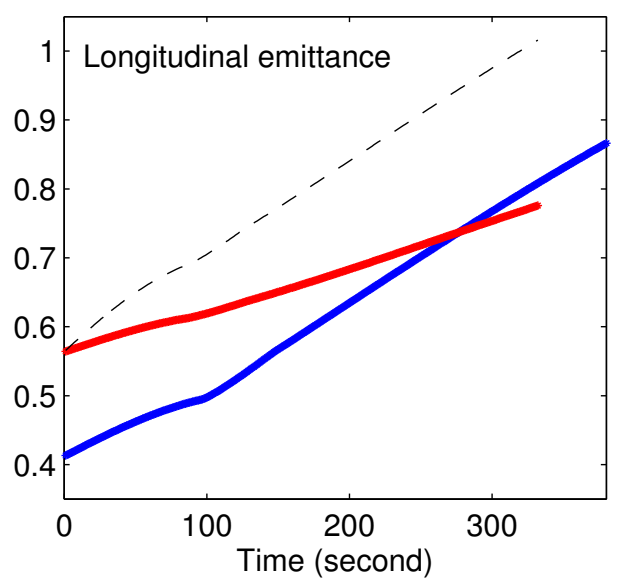

Figure 4: On the left side is the bunch length and longitudinal emittance in ramp, for 16086 in Run11, and 16998 in Run12, both fills have bunch intensity about $1.5 \times 10^{9}$. On the right side is the IBS simulation, 16086 of Run11 with the initial transverse emittance of $9 \pi \mu m$, and 16998 of Run12 with $18 \pi \mu m$. In addition to the different initial longitudinal and transverse emittance, the ramp time, the RF voltage, and the lattice are different for Run11 and Run12. The black dashed line is the simulation of 16998 of Run12, with the initial transverse emittance of $9 \pi \mu m$ (used for Run11), instead of $18 \pi \mu m$ (used for Run12). 
In Table 2, the detailed comparison of 16086 of Run11 and 16998 of Run12 is given, where the end transverse emittance (at flatop) due to the IBS effect is also shown.

\begin{tabular}{cccc}
\hline \hline Fill & & 16086 & 16998 \\
& & Run11 & Run12 \\
\hline Ramp time, $t$ & $s$ & 380 & 330 \\
RF voltage at flatop, $V$ & $k V$ & 306 & 253 \\
Lattice & & Au104 & CuAu12 \\
Bunch intensity, $N_{b h}$ & $10^{9}$ & 1.50 & 1.51 \\
Initial longitudinal emittance, $\epsilon_{\ell}$ & $e V s / n$ & 0.41 & 0.56 \\
End longitudinal emittance, $\epsilon_{\ell}$ & $e V s / n$ & 0.87 & 0.77 \\
Initial transverse emittance, $\epsilon_{N}$ & $\pi \mu m$ & 9.0 & 18.0 \\
End transverse emittance, $\epsilon_{N}$ & $\pi \mu m$ & 11.9 & 19.3 \\
\hline \hline
\end{tabular}

Table 2: Comparison of high intensity Au beam in ramp of 16086 in Run11 and 16998 in Run12, with same bunch intensity. The initial longitudinal and transverse emittance are different in these two runs (also the ramp time, the $\mathrm{RF}$ voltage, and the lattice are different).

In Run11, the large longitudinal emittance growth from injection to the rebucketing was of interest, where it is, typically, $0.3 \mathrm{eVs} / \mathrm{n}$ at the injection, $0.4 \mathrm{eVs} / n$ at the accramp, then it is $>0.9 \mathrm{eVs} / n$ at the flatop. Now in Run12, the strong growth of the longitudinal emittance is alleviated because of the larger initial longitudinal emittance of EBIS beam at the RHIC injection, and more importantly, the large transverse emittance with high intensity beams. In Run12, the longitudinal emittance at the flatop is smaller than $0.8 \mathrm{eVs} / \mathrm{n}$.

Smaller longitudinal emittance is helpful for the RF rebucketing. The required $197 \mathrm{MHz}$ RF voltage is not high in Run12, and the lower RF voltage in the rebucketing implies smaller momentum spread in store, which reduces the beam loss due to the dynamic aperture limit. In general, small longitudinal emittance is desired, either with the $197 \mathrm{MHZ}$ rebucketing, or when 56 $\mathrm{MHz}$ cavity becomes available. 
For RHIC luminosity improvement, the transverse emittance dependence on intensity is perhaps more important. With current EBIS and injector settings, the stochastic cooling's power requirement is tighter to get a comparable integrated luminosity at RHIC.

With these, the EBIS and the injection chain are clearly a focus of the improvement. This report, with the understanding and questions raised in Run12, might be useful.

On the other hand, the current situation, i.e., put 4 Booster loads into 1 RHIC bunch, has a potential to achieve even higher intensity in RHIC. Since the estimated transverse emittance dependence is (only) proportional to bunch intensity, and there is a quadratic relation of the intensity with the luminosity, a naive thinking is to exhaust this direction in the RHIC luminosity push, together with the injector improvement.

\section{References}

[1] S.Y. Zhang, "A Luminosity Model of RHIC Gold Runs", C-A/AP/438, BNL, 2011.

[2] S.Y. Zhang and C. Montag, http://www.cadops.bnl.gov/AP/Meetings// RHIC_Monday_meetings/2011/October/SYZ111016.pdf 